\title{
Klebsiella pneumoniae liver abscess complicated by endogenous endophthalmitis: the importance of early diagnosis and intervention
}

\section{Clinical record}

A 51-year-old man presented to the Royal Victorian Eye and Ear Hospital with 3 days of progressive visual loss and pain in the left eye, and a 6-hour history of painless visual loss in the right eye. He reported a l-week history of fever, night sweats, sore throat and a non-productive cough. The man was systemically well, with no features of sepsis or abdominal pain.

His only medical history was for hypercholesterolaemia. He was Malaysian, but had lived in Australia for 10 years. He had recently travelled to Malaysia and Vietnam.

Visual acuity (VA) was hand movements in the left eye and $6 / 12$ in the right. He had a left relative afferent pupillary defect and bilateral hypopyon. Vitritis limited posterior segment examination. The left eye had lid swelling, conjunctival chemosis, proptosis, and computed tomography (CT) showed evidence of scleritis (Figure, A).

White cell count $\left(26 \times 10^{9} / \mathrm{L}\right.$; reference interval [RI], 4.0-11.0 $\times$ $10^{9} / \mathrm{L}$ ) and C-reactive protein levels (199 mg/L; RI <5 mg/L) were both elevated; liver function tests were deranged, with evidence of cholestasis. Liver ultrasonography revealed a $5.3 \mathrm{~cm}$ abscess in segment VII. A CT scan showed two abscesses: a $5.0 \times 5.7 \mathrm{~cm}$ abscess in segments V/VIII and a $3.3 \times 5.1 \mathrm{~cm}$ abscess in segment VII, with cholelithiasis and segmental thrombosis of the right hepatic vein (Figure, B). The results of blood cultures were negative.

$V A$ in the right eye deteriorated to counting fingers over a 24-hour period, and endogenous Klebsiella pneumoniae endophthalmitis was suspected. The patient received systemic ceftriaxone; bilateral intravitreal vancomycin, ceftazidime and dexamethasone; and oral and topical corticosteroid therapy. $K$. pneumoniae was cultured from ultrasound-guided drainage of one liver abscess and from urine. Repeated vitreous samples included polymorphonuclear leukocytes, but no bacteria could be cultured. Hepatic vein thrombosis was treated with therapeutic enoxaparin and, later, rivaroxaban

Pars plana vitrectomy (PPV) was performed in the right eye 2 days after presentation, and a superotemporal subretinal abscess was noted. Vision initially improved to $6 / 24$, but deteriorated to hand movements 2 days after the operation, presumably following rupture of the abscess. A second PPV was performed, and the abscess was drained. A rhegmatogenous retinal detachment was managed intraoperatively with

Phoebe P Moore BMed, MMed

Gerard F McGowan MB ChB, FRCOphth

Sukhpal S Sandhu MD, FRANZCO

Penelope J Allen MBBS, FRANZCO

The Royal Victorian Eye and Ear Hospital, Melbourne, VIC.

pjallen@

melbourneretina. com.au

doi: 10.5694/mjal5.00107 doses and the left eye five doses of intravitreal vancomycin ceftazidime and dexamethasone.

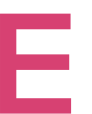
ndophthalmitis refers to inflammation of the intraocular space, and is predominantly of infectious aetiology. It is usually exogenous and may complicate intraocular surgery, penetrating trauma or corneal ulceration. Around $2 \%-8 \%$ of cases occur via endogenous spread, often in the context of immunosuppression, diabetes or injecting drug use. ${ }^{1}$

The left eye underwent a two-stage PPV, with the first surgery 4 days after presentation. An inferior subretinal abscess was drained during the second PPV (Figure, C and D). An associated rhegmatogenous retinal detachment was treated with endolaser and silicone oil.

Both eyes settled well (Figure, $\mathrm{E}$ and $\mathrm{F}$ ). The retinae remained attached bilaterally under oil, and best-corrected VA had improved to $6 / 12$ in the right eye and $6 / 24$ in the left eye at the 1-month follow-up.
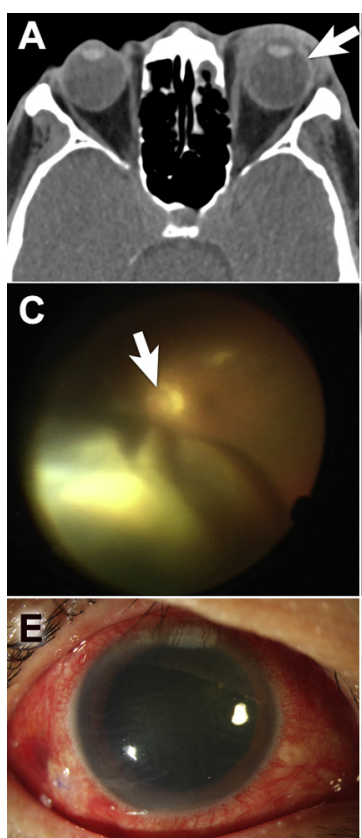

$A, B$ : Intraoperative photographs of the posterior segment of the eye showing retinal detachment with a subretinal abscess in the right eye $(\boldsymbol{A})$ and a subretinal abscess in the left eye $(B)$; the white arrows indicate the optic nerves. C: Computed tomography (CT) image of the orbits at presentation, showing left-sided proptosis with associated inflammation of the sclera (white arrow) and periorbital soft tissues. D: Abdominal CT scan, showing a $5.0 \times 5.7 \mathrm{~cm}$ abscess (white arrow) in segments V/VIII of the liver. $E$, F: Slit lamp photos of the right $(E)$ and left $(F)$ eyes 13 days after initial presentation.
In Australia, fungal organisms cause $65.9 \%$ of endogenous endophthalmitis, while gram-negative organisms cause $19.5 \%{ }^{1}$ In East Asia, there is an increasing incidence of disease caused by gram-negative organisms, with Klebsiella pneumoniae now the most common cause of endogenous endophthalmitis..$^{2-4} \mathrm{~K}$. pneumoniae is a known cause of pyogenic liver abscess in southeast Asia, 
with metastatic spread of infection occurring in $3.5 \%-20 \%$ of cases and endogenous K. pneumoniae endophthalmitis (EKPE) in $3 \%-7.8 \%$ of cases. ${ }^{3}$

EKPE has an extremely poor prognosis. ${ }^{2-5}$ Retinal damage occurs rapidly following irreversible necrosis of the retinal photoreceptor layer and the associated subretinal abscess formation. ${ }^{1-7}$ Final visual outcomes of hand movements or worse are reported in $66 \%-78 \%$ of cases, ${ }^{3,7}$ no perception of light in $57.8 \%-62 \%,{ }^{7,8}$ and evisceration or enucleation in $26.8 \%-75 \%$ of patients; ${ }^{1,8}$ in one report, three cases of EKPE required enucleation despite appropriate intensive antibiotic treatment. ${ }^{6}$

The poor prognosis is exacerbated by delayed diagnosis, particularly in patients who, because of overwhelming systemic illness, are unable to describe visual symptoms. ${ }^{2}$ Further significant predictors of a worse prognosis include poor initial visual acuity ${ }^{3}$ and the presence of hypopyon at presentation. ${ }^{8}$

In rare cases, EKPE may be the first manifestation of K. pneumoniae infection. ${ }^{3}$ EKPE is usually unilateral, but bilateral ocular involvement is reported in up to $26 \%$ of patients. ${ }^{1}$ Diabetes is a risk factor for EKPE. ${ }^{2,5}$ There is no general consensus about the most appropriate treatment for EKPE.

It has been suggested that pars plana vitrectomy (PPV) reduces intraocular bacterial and inflammatory load in EKPE and aids the penetration of intravitreal antibiotics. ${ }^{2,6}$ The best visual outcomes are seen in patients who undergo surgery early. ${ }^{4}$ In a Korean report about seven patients (10 eyes) with EKPE, early PPV was performed on eight eyes and delayed PPV on two. Operative findings included extensive retinal necrosis with subretinal abscess formation and dense vitritis. Five eyes maintained a final VA of counting fingers or better, VA in two eyes improved to 6/19 and 6/38; no enucleations or eviscerations were required. ${ }^{2}$ Another group described a patient who had PPV within 8 hours of the onset of EKPE symptoms; VA had improved from $6 / 120$ to $6 / 6$ at the 12 -month follow-up. ${ }^{9}$ These results
Lessons from practice

- Endogenous Klebsiella pneumoniae endophthalmitis (EKPE) carries an extremely poor visual prognosis.

- In patients admitted to general hospitals with K. pneumoniae liver abscess, a high index of suspicion of EKPE is recommended, and early referral to ophthalmology services advised.

- Early surgical intervention in EKPE can salvage vision in this otherwise devastatingly blinding disease.

support the role of early PPV in the management of EKPE.

A series of five patients had previously been treated for EKPE at the Royal Victorian Eye and Ear Hospital. All were treated with intravenous and intravitreal antibiotics. Four of the five patients required enucleation or evisceration, and the fifth became phthisical. Our patient, the sixth case, presented early in the course of disease, and was treated with systemic and intravitreal antibiotics, as well as with early PPV. In contrast to the preceding cases, the visual outcome was excellent.

The incidence of $K$. pneumoniae liver abscess in the Asia-Pacific region is increasing. Early ophthalmology referral for patients with suspected or proven K. pneumoniae liver abscess is recommended. In patients with known EKPE, early PPV should be performed in conjunction with intensive systemic and repeated intravitreal antibiotic and steroid therapy to reduce intraocular bacterial and inflammatory load, and to aid the penetration of intravitreal antibiotics to the subretinal focus of infection. This may increase the chances for rescuing the eye and improving the visual outcome.

Competing interests: No relevant disclosures.

(c) 2015 AMPCo Pty Ltd. Produced with Elsevier B.V. All rights reserved.

References are available online at www miacom.au. 
1 Connell P, O’Neill E, Fabinyi D, et al. Endogenous endophthalmitis: 10-year experience at a tertiary referral centre. Eye 2011; 25: 66-72.

2 Yoon $\mathrm{H}$, Lee S, Sohn J, et al. Result of early vitrectomy for endogenous Klebsiella pneumoniae endophthalmitis. Retina 2003; 23: 366-370.

3 Sheu S, Kung Y, Wu T, et al. Risk factors for endogenous endophthalmitis secondary to Klebsiella pneumoniae liver abscess: 20-year experience in southern Taiwan. Retina 2011; 31: 2026-2031.

4 Lee $\mathrm{S}, \mathrm{Um} \mathrm{T}$, Joe $\mathrm{S}$, et al. Changes in the clinical features and prognostic factors of endogenous endophthalmitis: fifteen years of clinical experience in Korea. Retina 2012; 32: 977-984.

5 Chen Y, Kuo H, Wu P, et al. A 10-year comparison of endogenous endophthalmitis outcomes: an East Asian experience with Klebsiella pneumoniae infection. Retina 2004; 2: 383-390.

6 Sridhar J, Flynn H, Kuriyan A, et al. Endophthalmitis caused by Klebsiella species. Retina 2014: 34: 1875-1881.

7 Wong J, Chan T, Lee H, et al. Endogenous bacterial endophthalmitis: an East Asian experience and a reappraisal of severe ocular affliction. Ophthalmology 2000; 107: 1483-1491.

8 Ang M, Jap A, Chee S. Prognostic factors and outcomes in endogenous Klebsiella pneumoniae endophthalmitis. Am J Ophthalmol 2011; 151: 338-344.

9 Ishii K, Hiraoka T, Kaji Y, et al. Successful treatment of endogenous Klebsiella pneumoniae endophthalmitis: a case report. Int Ophthalmol 2011; 31: 29-31. 